\title{
Three Dimensional Printing of Metals and Ceramics Graded Dendrites for Energy and Material Flows Modulation
}

\author{
Soshu Kirihara \\ Osaka University, kirihara@jwri.osaka-u.ac.jp
}

\begin{abstract}
Artificial ceramic dendrites were successfully fabricated by three dimensional printing of stereolithography. The ceramics nanoparticles were dispersed in to photo sensitive liquid resins were spread uniformly by a mechanical knife edge. The cross sectional pattern was formed through illuminating visible laser. The high resolution image could be achieved by a digital micro mirror device. The solid micro structures were built by stacking these patterns. The formed precursors with dendrite structures were dewaxed and sintered in air atmosphere. By utilizing stereolithography, micro photonic crystals with diamond lattice structures composed of alumina or copper were fabricated. Subsequently, solid electrolyte dendrites of yttria stabilized zirconia with spatially ordered porous structures were fabricated for fuel cell miniaturizations. Moreover, artificial bones of hydroxyapatite scaffolds with dendritic lattice structures were modeled successfully to realize excellent biological compatibilities. Graded porous structures in the artificial bones were processed as novel tissue engineering.
\end{abstract}

Keywords: Micro Stereolithogarphy, Graded Dendrite, Photonic Crystal, Porous Electrode, Biological Scaffold

\section{Introduction}

Three dimensional (3D) printing of micro pattering stereolithography had been developed successfully as an additive manufacturing $(\mathrm{AM})$ process to create electromagnetic devices of photonic crystals. The artificial crystals with periodic arrangements of dielectric media can reflect the electromagnetic wave perfectly by Bragg diffraction [1,2]. Forbidden gaps are exhibited in the transmission spectra. Through introductions of air cavities into the periodic lattices, the electromagnetic waves having specific wavelengths can resonate with structural defects. Localized modes of transmission peaks are formed in the band gap. The alumina micro lattices with diamond structures were fabricated to control terahertz waves. The terahertz waves are expected to detect micro cracks in material surfaces and structural defects in electric circuits by fine wave interferences, and to analyze cancer cells in human skins and toxic bacteria in natural foods through the higher frequency excitations. Subsequently, porous electrodes were processed by micro stereolithography to be applied for energy harvesting. Solid oxide fuel cells (SOFCs) investigated as novel generation systems of electric powers with high efficiencies in energy conversion circulations. Yttria stabilized zirconia (YSZ) with high ion conductivities for incident oxygen is widely adopted material for solid electrolyte anodes as the SOFC components [3]. Solid electrolyte dendrites composed of YSZ spatial lattice structures with various coordination numbers were printed in 3D to increase the surface areas of the electrode for fuel cell miniaturizations. In the dendritic lattice structure, stress distributions and fluid flows were simulated and visualized by using finite element methods. These new computer aided designs, manufactures and evaluations have been established and optimized to create micro components of various ceramics in our investigation group. Moreover, artificial bones composed of hydroxyapatite (HAP) scaffolds with dendritic lattices of ordered porous structures were formed to realize excellent biological compatibilities [4]. The micro ceramics rods were arranged periodically to create lattice arrangements with four coordination number, and these aspect ratios were modulated continuously to realize biomimetic graded structures. Fine hydroxyapatite microstructures without cracks or pores were obtained.These results show that the stereolithographic technique is a novel technology that can be used to fabricate bio-scaffolds. The successfully manufactured bone substitute and the strictly designed bioscaffold structures can be used to promote appropriate biochemical reactions and body fluid circulation. 


\section{Experimental Procedure}

The stereolithography system illustrated schematically as shown in Fig. 1. The designed graphic data was converted into the stereolithographic file format through polyhedral approximations. The solid model was sliced into the cross sectional numerical data sets to input the stereolithography equipment. Photo sensitive acrylic resins dispersed with nanoparticles were fed over a substrate from a dispenser nozzle. The highly viscous resin paste was fed with controlled air pressure, and spread uniformly by a mechanical knife edge. The thickness of each layer was controlled to $10 \mu \mathrm{m}$. The cross sectional 2D pattern was formed through illuminating visible laser of $405 \mathrm{~nm}$ in wavelength on the resin surface. The high resolution image could be achieved by applying a digital micro mirror device (DMD) and an objective optical lens. The DMD is an optical element assembled by micro mirrors of $14 \mu \mathrm{m}$ in edge length. The tilting of each tiny metal mirror can be controlled according to the cross sectional data. The solid micro structures were built by stacking these patterns layer by layer. In order to avoid deformation and cracking during dewaxing, careful and systematic investigation for the heat treatment was required.

The photonic crystals with the diamond lattices were designed by using the graphic application (Think Design: Toyota Caelum, Japan). The lattice constant was $1 \mathrm{~mm}$ in dimension. The whole structure was $6 \times 6 \times 2 \mathrm{~mm}^{3}$ in size, consisting of $6 \times 6 \times 2$ unit cells. The aspect ratio of the dielectric lattices was designed to be 1.5. The graphic data was transferred to the stereolithographic equipment. In the fabrication process, photo sensitive acrylic resin dispersed with alumina particles of $170 \mathrm{~nm}$ in diameter at 40 vol. \% was used. The precursors with diamond structures were heated at various temperatures from 100 to $600{ }^{\circ} \mathrm{C}$, while the heating rate was set at $1.0{ }^{\circ} \mathrm{C} / \mathrm{min}$. The dewaxing process was observed in respect to the weight and color changes. Nanometer sized alumina particles could be sintered at $1500{ }^{\circ} \mathrm{C}$. The

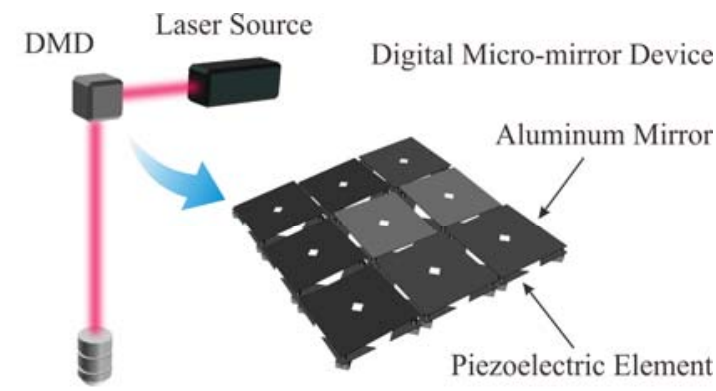

Objective Lens

\section{EXPOSURE}

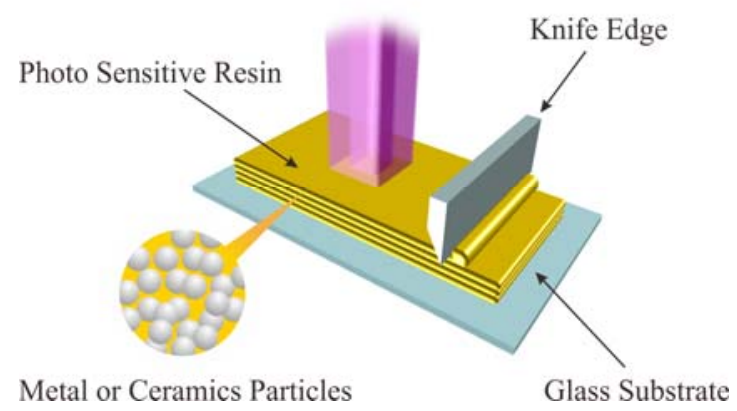

Metal or Ceramics Particles

Fig. 1 A schematically illustrated three dimensional printing (3D) of a micro patterning stereolithography system (D-MEC: SI-C1000, Japan). Fine micro images are exposed by using a digital micro mirror device on a photo sensitive resin with homogenious dispersions of various ceramics nanoparticles.

heating rate was $8.0^{\circ} \mathrm{C} / \mathrm{min}$. The density of the sintered sample was measured by the Archimedes method. The microstructure of the lattices was observed by using scanning electron microscopy (SEM). The transmittance and the phase shift of incident terahertz waves were measured by using terahertz wave time-domain spectroscopy (AISpec: J-Spec2001, Japan). Measured terahertz wave properties were compared with electromagnetic band diagrams of diamond structures theoretically calculated by a plane wave expansion method. Intensity profiles of the electric field were simulated by using transmission line modeling (TLM) method program (Flomerics: Micro Stripes, Germany).

The solid electrolyte dendrites with spatial lattice structures were designed by using the computer graphic application. The dendrite lattices of 1.16 in aspect ratio can be considered to exhibit the higher reaction efficiencies and gas transmittances according to Nernst equation. In the optimized dendrite structure, the diameter and length of YSZ rods were decided as 92 and $107 \mu \mathrm{m}$, respectively. The lattice constant was $250 \mu \mathrm{m}$. Photo sensitive acrylic resin dispersed with YSZ particles of 60 and $100 \mathrm{~nm}$ in first and second diameters at $30 \mathrm{vol}$. \% were used for the stereolithography. The formed precursors with dendrite structures were heated at various temperatures from 100 to $600{ }^{\circ} \mathrm{C}$ while the heating rate was $1.0{ }^{\circ} \mathrm{C} / \mathrm{min}$. The dewaxing process was observed in respect to the weight and color changes. The YSZ particles could be sintered at $1500{ }^{\circ} \mathrm{C}$ for $2 \mathrm{hs}$. The heating rate was $8.0^{\circ} \mathrm{C} / \mathrm{min}$. The density of the sintered sample was measured by using Archimedes method. The ceramic microstructures were observed by a digital optical microscope and scanning electron microscopy. In the lattice dendrites, fluid flow velocities and pressure stress distributions were simulated and visualized by a finite volume method (FVM) application (Ansys: Cybernet Systems, Japan). In these simulations, no slip surface conditions were assumed.

The biological scaffolds with four coordinate rods were connected to form diamond lattice structures with a periodic length of $1.8 \mathrm{~mm}$. The aspect ratios were adjusted in the range 1.2 to 2.0 in the scaffold models to control the porosity in the range from 50 to $80 \mathrm{vol}$. \%. Photosensitive acrylic resins with hydroxyapatite powder at $40 \mathrm{vol} \%$ were used for the materials of slurry. The fabricated precursors were dewaxed at $600{ }^{\circ} \mathrm{C}$ for $2 \mathrm{hs}$ at a heating rate of $1.0^{\circ} \mathrm{C} / \mathrm{min}$ and sintered at $1250{ }^{\circ} \mathrm{C}$ for $2 \mathrm{hs}$ at a heating rate of $5.0^{\circ} \mathrm{C} / \mathrm{min}$ in air. The hydroxyapatite porous body form and microstructure were observed using an digital optical microscope (DOM) and a scanning electron microscope (SEM), respectively. The sintered densities were measured by the Archimedes' method. Fluid circulation in the ordered and graded porous models was visualized with the fluid dynamic solver (Ansys: Cybernet Systems, Japan). Flow directions and velocities were calculated in spatial grids in the scaffold models through the finite element method (FEM). The simulation parameters were used to investigate the behavior of the blood flow in bone substitute. The fluid phase was represented as an incompressible Newtonian fluid with a viscosity of $1.45 \times 10-3 \mathrm{~Pa} \cdot \mathrm{s}$. The inlet velocity applied to the scaffolds was constant at $0.235 \mathrm{~mm} / \mathrm{s}$, and the pressure was zero at the outlet. 


\section{Results and Discussion}

The acryl photonic crystal of sintering precursor with the diamond structure with alumina nanoparticles dispersions was fabricated by using the stereolithography. The spatial resolution was approximately $0.5 \%$. Through the dewaxing and sintering processes, ceramic diamond structures were successfully obtained as shown in Fig. 2. The deformation and cracking were not observed. The lattice constant of the diamond structure is $375 \mu \mathrm{m}$. The linear shrinkage was $24 \%$. It is possible to obtain the uniform shrinkage by designing an appropriate elongated structure in the vertical direction for compensation to the gravity effect. The relative density reached $97.5 \%$. The measured band gap frequencies were compared with calculation results by the plane wave expansion method. The measured frequency ranges of opaque regions corresponded to the calculation. According to the photonic band diagram, it was demonstrated that a complete photonic band gap opened between $0.40 \mathrm{THz}$ and $0.47 \mathrm{THz}$. The modified diamond structure was fabricated by the three dimensional printing as shown in Fig. 3 . The two and three periods lattice structures were arranged on the right and left side of a plane defect, respectively. The terahertz wave was transmitted from the left side to the right direction. In the measured transmission spectrum, one localized mode peak was observed in the band gap at the frequencies of $0.42 \mathrm{THz}$. The measured band gap region and peak frequency of the localized mode were compared with calculations by TLM method. They were in good agreement. The electric field distribution in the twinned diamond lattices was simulated. Figure 3 shows cross sectional images of the distributions. In this image, the red area indicates that the electric field intensity is high, whereas blue and green area indicates it is low. Incident terahertz wave is resonate and localized in the plane defect region between twinned diamond lattices. The amplified electromagnetic wave by multiple reflections can transmit through the photonic crystal. Therefore, the transmission peak will be formed in the band gap. On the right side of the sample, the radiation pattern shows the plane wave expansion. The micro photonic crystal with the twinned ceramic lattice of the diamond structure can be applied to a terahertz beam emitters.

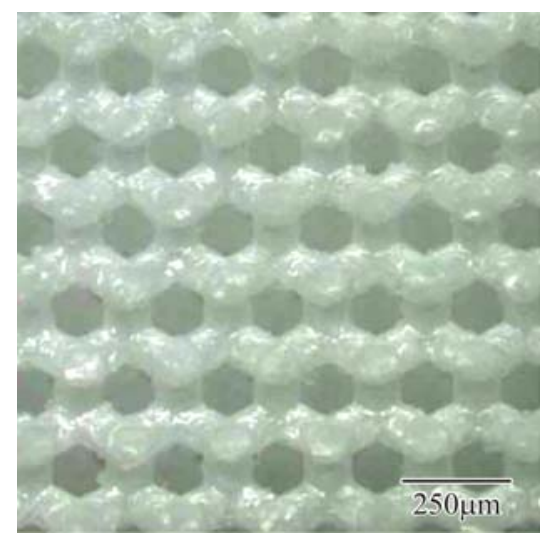

Fig. 2 An alumina photonic crystal formed by using stereolithography.

Incident Direction $\longrightarrow$
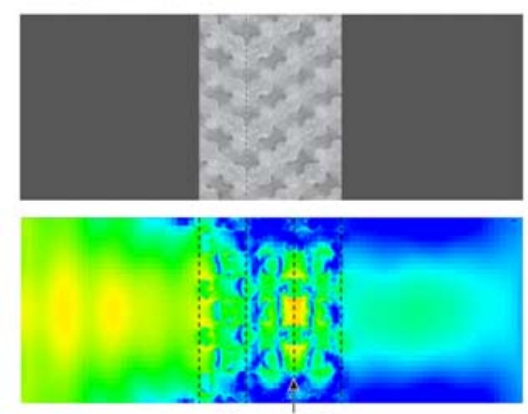

Plane Defect

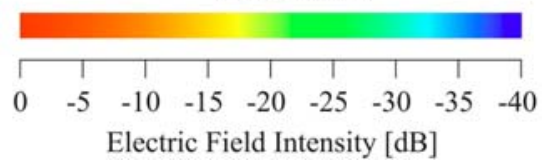

Fig. 3 A modified photonic crystal with a twinned lattice structure.

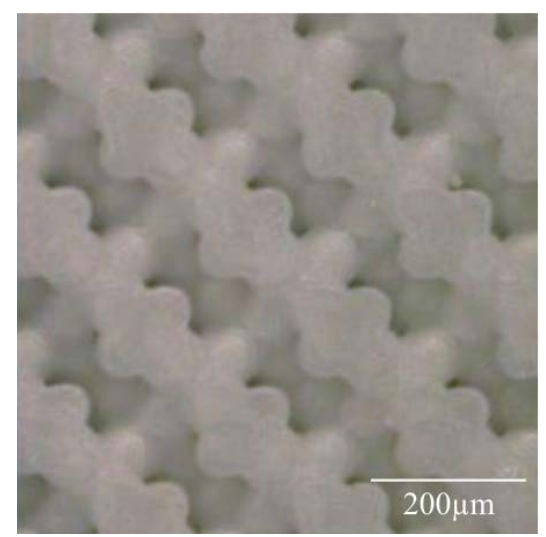

Fig. 4 A sintered dendrite lattice of yttria stabilized zirconia electrolyte.

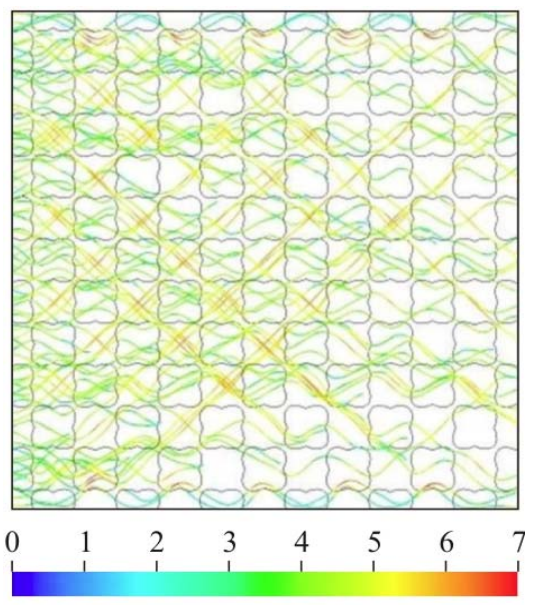

Fluid Flow Velocity [m/s]

Fig. 5 A distribution of fluid flow velocities in the YSZ dendrite.

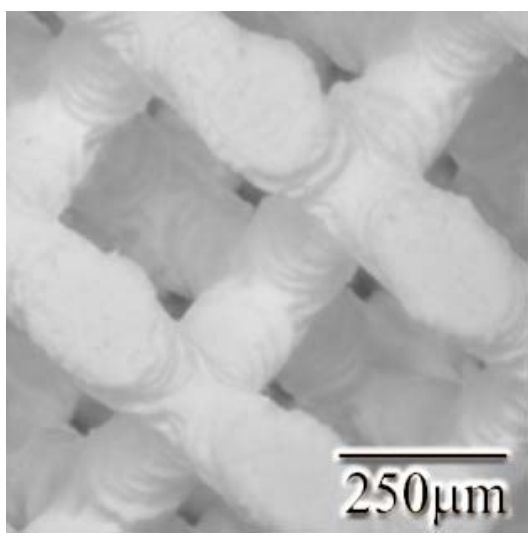

Fig. 6 A hydroxyapatite scaffold of ordered lattice and porous structures.

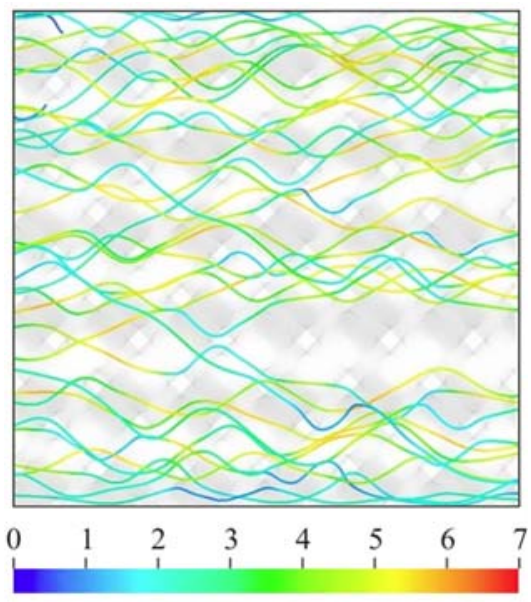

Fluid Flow Velocity $[\mathrm{m} / \mathrm{s}]$

Fig. 7 The fluid flow velocities in the HAP dendrite structure. 
The dendritic acryl lattice structures with YSZ particles dispersions were processed exactly by using the stereolithography. The spatial resolution was approximately $1.0 \%$. In the microstructure of the composite lattice, the nanometer sized YSZ particles were dispersed homogeneously in the acryl matrix. Figure 4 shows the sintered solid electrolyte dendrite with the YSZ micro lattice structure. The deformation and cracking were not observed. The linear shrinkages on the both horizontal and vertical axis were $32 \%$. The volume fraction of the air gaps was $50 \%$ by the open paths. In the other previous investigations, the porous electrodes were formed by sintering the YSZ surly with polystyrene particles dispersion. Therefore, it is difficult to realize the prefect opened pores structures with the higher porosity over $40 \%$ in volume fraction. The dense microstructure of the YSZ lattice was obtained. The average grain size was approximately $4 \mu \mathrm{m}$. The relative density reached at $95 \%$. Micrometer sized cracks or pores were not observed. The

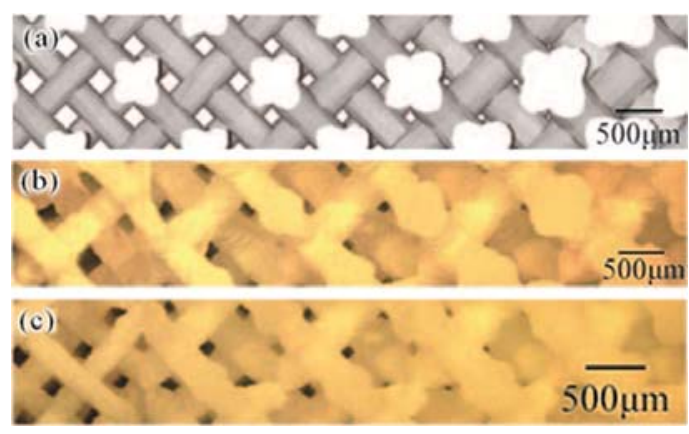

Fig. 11 Graded lattices of designed model (a), acrylic lattices with hydroxylapatite particles by stereolithography (b) and sintered scaffold (c).

obtained dense YSZ lattice structure will be exhibit the higher performances in mechanical properties as the porous electrodes of the solid electrolyte dendrites. The fluid flow velocities were visualized by using the FVM method as shown in Fig. 5 . Continuously curved lines indicate the fluid distributions along the vector directions of flow velocities. All air paths were opened for outsides and connected with each other in the YSZ dendrite lattice structures. The fluid flows can transmit the one direction smoothly. The pressure stress distributions in the dendrite were visualized. The fluid pressures were gradually distributed for flow direction, and the localization of the stress was not observed. The fabricated solid electrolyte dendrites with lattice structures can be considered to have higher performances as novel ceramic electrodes in near future.

The acrylic resin structures incorporating the hydroxyapatite particles were fabricated precisely following the designed model. The part accuracies of the green bodies were measured under $50 \mu \mathrm{m}$ in size difference. These were caused during the resin polymerization at the micro pattern exposures. The hydroxyapatite particles were dispersed homogeneously in the acrylic resin matrix. A dense ceramic structure was formed as shown in Fig. 6. The linear shrinkage along the horizontal and vertical axes was 23 and $25 \%$, respectively. The porosity of the ordered structure was about $70 \%$. The relative density of the sintered body reached $98 \%$. The microstructure was affected by grain size distribution of hydroxyapatite, but it has been uniformly sintered without abnormal grain growth. The visualized fluid flow behaviors in the ordered porous and lattice structures are shown in Fig. 7 as cross sectional graphics. The relation between the fluid velocity and cell deformation were reported, and the high fluid velocity area in the scaffold is subjected to a shear stress which can assume the cell attachment difficulty. An isotropic distribution of flow rates was observed in the formed scaffold. And, this structure is insusceptible to a shear stress. Homogeneous tissue regeneration will be promoted through geometrical modification of the scaffold models. Hence, this is invaluable simulation for the tissue engineering before confirmative osteoconduction in vivo. The designed scaffold model with the graded porous structure is shown in Fig. 8. The porosity is distributed gradually through modulations of the aspect ratio in the 4 coordination number lattices as shown in Fig. 9-(a). The acryl scaffolds with hydroxyapatite particles is shown in Fig. 9-(b). The sintered scaffold model of the hydroxyapatite ceramic with the graded lattices is shown in Fig. 9-(c). The linear shrinkage ratios for horizontal and vertical axis were 23 and $25 \%$, respectively. The smaller lattice structures could be obtained effectively through the controlled body

\section{Conclusion}

By using micro pattering stereolithography, micrometer order photonic crystals with a diamond structure composed of alumina dispersed acrylic resin were fabricated. The alumina photonic crystal formed a complete band gap at the terahertz region. Localized modes were obtained by introducing a plane defect between twinned lattices. It is expected that the photonic band gap structures can be applied to control terahertz waves. Solid electrolyte dendrites with yttria stabilized zirconia lattices were fabricated for anode electrodes of solid oxide fuel cells. Acryl precursors including ceramic particles were formed. Thorough careful optimization of process parameters in dewaxing and sintering, dense ceramic micro components were obtained. These solid electrolyte dendrites with opened air path networks exhibited effective transmission properties of fluid flows. These novel ceramic electrodes have potentials to contribute for developments of compact fuel cells. Hydroxyapatite biological scaffolds with geometrical variations were fabricated. The sintering body of scaffolds could precise reproduce exactly ordered porosities. Flow simulations will be powerful tools before time consuming clinical test. Structural modifications to form natural graded structures may help in realizing effective regeneration in implanted tissue engineering.

\section{References}

1. E. Yablonovitch, Physical Review Letters, 58 (1987) 2059.

2. S. Kirihara, Y. Miyamoto, International Journal of Applied Ceramic Technology, 6 (2009) 41.

3. N. Minh, Journal of the American Ceramic Society, 76 (1993) 563.

4. S. Yang, K. F. Leong, Z. Du, C. K. Chua, Tissue Engineering, 8 (2002) 1. 